\title{
QUANTIFICAÇÃO DE ÁCIDO ALFA-LINOLÊNICO EM CAULES E FOLHAS DE LINHO (Linum usitatissimum L.) COLHIDOS EM DIFERENTES ESTÁGIOS DE DESENVOLVIMENTO
}

\author{
Quantification of alpha-linolenic acid in stems and leaves of flax (Linum usitatissimum L.) \\ harvested in different stages of development
}

\author{
Ana Carolina de Aguiar', Sílvia Cristina de Aguiar², Marcela Boroski³, Nilson Evelázio de Souza², \\ Makoto Matsushita², Jesuí Vergílio Visentainer ${ }^{2}$
}

\begin{abstract}
RESUMO
O objetivo deste estudo foi quantificar o ácido alfa-linolênico [LNA, 18:3 (n-3)], avaliar a composição centesimal das folhas e caules de linho (Linum usitatissimum L.) submetidos à secagem e colhidos em diferentes estágios de desenvolvimento (40, 80 e 120 dias), e determinar o potencial antioxidante das folhas colhidas aos 80 dias através do teste com o radical DPPH. As folhas obtiveram maiores teores de cinzas, proteína e lipídios totais em relação aos caules. Tanto as folhas quanto os caules apresentaram razões de AGPI/AGS e n-6/n-3 dentro dos valores considerados adequados para a alimentação. Os caules colhidos nos diferentes tempos não apresentaram diferenças significativas $(\mathrm{P}<0,05)$, quanto ao teor de LNA. As folhas colhidas aos 80 dias apresentaram a maior concentração de LNA, correspondendo a 1,262,36 mg/100g de folhas desidratadas. Os diferentes extratos (metanólico, butanólico, acetato e aquoso) foram eficientes na inibição do radical DPPH, com destaque para as frações butanólica e acetato, sendo os valores de $\mathrm{IC}_{50}$ de aproximadamente $42 \mathrm{ppm}$ para as duas frações. Tais resultados evidenciaram a atividade antioxidante e potencial nutritivo das folhas e caules de linho para futuro uso na alimentação animal e humana.
\end{abstract}

Termos para indexação: Ômega-3, ácidos graxos, atividade antioxidante, DPPH.

\begin{abstract}
The objective of this study was to quantify the alpha-linolenic acid [LNA, 18:3 (n-3)] and to evaluate the proximate composition of leaves and stems of flax (Linum usitatissimum L.) dried and harvested at different stages of development (40, 80 and 120 days), and to determine the antioxidant potential of the leaf harvested at 80 days using the test of DPPH radical. The leaves had higher levels of ash, protein and total lipids when compared to the stems. Both the leaves as the stems had ratios of PUFA/SFA and $\mathrm{n}-6 / \mathrm{n}-3$ within the values considered suitable for food. Stems in the different stages showed no significant difference $(\mathrm{P}<0.05)$ of LNA content. Leaves harvested at 80 days showed the highest concentration of LNA, corresponding to 1,262.36 mg/100g dried leaf. The different extracts (methanol, butanol, acetate and water) were efficient in the inhibition of DPPH radical, with emphasis on the butanolic and acetate fractions and the values of $\mathrm{IC}_{50}$ were approximately $42 \mathrm{ppm}$. These results highlight the nutritional potential and antioxidant activity of leaves and stems of flaxseed for future use in the animal and human feeding.
\end{abstract}

Index terms: Omega-3, fatty acids, antioxidant activity, DPPH.

(Recebido em 8 de agosto de 2009 e aprovado em 10 de março de 2010)

\section{INTRODUÇÃO}

O linho (Linum usitatissimum L.) é uma espécie que se destina à produção de fibras têxteis de alta qualidade e sua semente, a linhaça, é matéria-prima para produção de óleo e diversos produtos alimentícios (Morris, 2005).

De acordo com Kiss \& Souza (2000), o único cultivo de linho no Brasil reside em Guarani das Missões, no noroeste gaúcho, mantido por descendentes de imigrantes poloneses e alemães. As lavouras de linho já foram cultivadas no Brasil para a extração de fibras para o setor têxtil, hoje, porém, com a valorização do óleo de linhaça, seu objetivo também abrange a produção de sementes.

Sabe-se que a linhaça é uma das maiores fontes vegetais do ácido alfa-linolênico [LNA, 18:3 (n-3)], alcançando teores de até 55\% em sua fração lipídica (Carter, 1993). O LNA é um ácido graxo poli-insaturado da série n-3 (AGPI n-3), precursor de importantes ácidos graxos de cadeia longa dessa série, como o ácido docosaexaenóico [DHA, 22:6 (n-3)] e o ácido eicosapentaenóico [EPA, 20:5 (n-3)], considerados fundamentais para a manutenção de um organismo saudável (Martin et al., 2005). Inúmeras pesquisas evidenciam que eles estão envolvidos em processos

\footnotetext{
1Universidade Estadual de Maringá/UEM - Avenida Colombo - 5790 - Campus Universitário Maringá - 87.020-900 - Maringá, PR - anacamga@gmail.com 2Universidade Estadual de Maringá/UEM - Maringá, PR

3Universidade Estadual de Maringá/UEM - Departamento de Química - Maringá, PR
} 
metabólicos, atividades cardiovasculares (Schaghy, 2000; Visentainer et al., 2000), redução da taxa de colesterol e do risco de doenças como artrite e câncer (Simopoulos, 1991).

Os ácidos graxos poli-insaturados são altamente suscetíveis à oxidação, sendo necessária a utilização de agentes antioxidantes, os quais são definidos por Bercker et al. (2004), como compostos que, quando presentes em pequenas concentrações comparadas a do substrato oxidável, reduz significativamente ou previne a oxidação dessas substâncias. Uma inúmera variedade de alimentos de origem vegetal é fonte de compostos que apresentam atividade antioxidante (Moure et al., 2001). Diversos estudos evidenciam a importância do consumo de alimentos que contenham substâncias com propriedades antioxidantes em sua composição para prevenção de doenças crônicas, como enfermidades cardiovasculares, câncer e desordens cerebrais degenerativas associados ao envelhecimento (Chu et al., 2000; Liyana-Pathirana \& Shahidi, 2006).

Pesquisas recentes vêm sendo realizadas com a suplementação de linhaça e/ou o óleo de linhaça em animais e produtos alimentícios, por exemplo, peixes (Visentainer et al., 2005; Souza et al., 2008), bovinos (Prado et al., 2008), leite (Silva et al., 2006). Nesses estudos, em geral, foram avaliadas a inclusão de LNA, conversão em ácidos graxos de cadeia longa, taxa de conversão e acúmulo de LNA.

Caules e folhas do linho são resíduos do processo de obtenção da linhaça e do seu óleo. Não foram encontrados, na literatura, estudos sobre a caracterização química do caule e da folha do linho durante o crescimento da planta. Objetivouse, neste estudo, quantificar o ácido alfa-linolênico, avaliar a composição centesimal das folhas e caules de linho colhidos em diferentes estágios de desenvolvimento, além de determinar o potencial antioxidante das folhas, a fim de fornecer subsídios para futuros estudos do uso do caule e folhas do linho na alimentação humana e animal.

\section{MATERIAL E MÉTODOS}

$\mathrm{O}$ experimento foi realizado na zona rural do município de Marialva - PR ( $23^{\circ}$ sul e $51^{\circ} 44^{\prime}$ oeste), no período de maio a julho de 2007. A altitude média é de $670 \mathrm{~m}$ e o clima é mesotérmico úmido, tipo Cfa (Köppen).

As parcelas foram compostas de seis fileiras de $4 \mathrm{~m}$ de comprimento, espaçadas de $0,50 \mathrm{~m}$. As plantas foram colhidas manualmente nos tempos 40, 80 e 120 dias após a germinação, sendo as folhas separadas manualmente dos caules e ambos submetidos à secagem em estufa com circulação de ar por 36 horas à $50^{\circ} \mathrm{C}$, trituradas em moinho de facas, armazenadas em embalagens de polietileno a $-18^{\circ} \mathrm{C} e$, posteriormente, submetidas às análises.
Análises da composição centesimal:

As análises dos teores de umidade, cinzas e proteína foram realizadas conforme técnicas de Cunnif (1998). Os lipídios totais foram extraídos segundo Bligh \& Dyer (1959), sendo todas as análises realizadas em triplicata. Todos os reagentes utilizados foram de padrão analítico.

Transesterificação e análise dos ésteres metílicos de ácidos graxos (EMAG):

A transesterificação nos lipídios totais foi realizada em triplicata segundo o procedimento de Hartman \& Lago (1973). Os EMAG (ésteres metílicos de ácidos graxos) foram separados em um cromatógrafo a gás 14-A (Shimadzu, Japão), equipado com coluna capilar de sílica fundida CP Sil-88 (50 m de comprimento, $0,25 \mathrm{~mm}$ de diâmetro interno e $0,25 \mathrm{~mm}$ de biscianopropil polisiloxano) e detector de ionização de chama. Os fluxos dos gases foram de $1,2 \mathrm{~mL} /$ min para o gás de arraste, $\mathrm{H}_{2}, 30 \mathrm{~mL} / \mathrm{min}$ para o gás auxiliar $\mathrm{N}_{2}$, e 30 e $300 \mathrm{~mL} / \mathrm{min}$ para os gases da chama $\mathrm{H}_{2}$ e ar sintético, respectivamente. A razão de divisão da amostra foi de 1:100. A temperatura da coluna foi elevada de $150^{\circ} \mathrm{C}$ a $185^{\circ} \mathrm{C}$ a uma taxa de $2^{\circ} \mathrm{C} / \mathrm{min}$, sendo então elevada para $225^{\circ} \mathrm{C}$ a $10^{\circ} \mathrm{C} / \mathrm{min}$, permanecendo nessa temperatura por $20 \mathrm{~min}$. As temperaturas do injetor e do detector foram $220^{\circ} \mathrm{C}$ e $230^{\circ} \mathrm{C}$, respectivamente. As injeções foram realizadas em duplicata e o volume foi de $2 \mathrm{~mL}$. As áreas dos picos foram determinadas utilizando-se um Integrador-Processador CG300 (Instrumentos Científicos CG). As identificações foram efetuadas através da comparação dos tempos de retenção de ésteres metílicos de padrões da Sigma (EUA) com os das amostras, e a quantificação dos ácidos graxos baseouse no método da normalização de área (Visentainer \& Franco, 2006), em que expressa-se a concentração dos mesmos em porcentagem relativa de lipídios totais.

Quantificação do ácido alfa-linolênico:

$\mathrm{O}$ ácido LNA existente nos lipídios totais foi quantificado em $\mathrm{mg} / \mathrm{g}$ de lipídios totais, utilizando o tricosanoato de metila (23:0) como padrão interno. Os cálculos foram realizados segundo método de Joseph \& Ackman (1992), conforme equação 1.

$$
M x=\frac{A_{x} x M_{p} x F_{c t}}{A_{p} x M_{a} x F_{c e a}}
$$

Onde:

$\mathrm{M}_{\mathrm{x}}$ : massa do ácido graxo $\mathrm{x}$ em $\mathrm{mg} / \mathrm{g}$ de lipídio;

$\mathrm{M}_{\mathrm{p}}$ : massa do padrão interno em mg;

$\mathrm{M}_{\mathrm{a}}^{\mathrm{p}}$ : massa da amostra de lipídios totais em $\mathrm{g}$;

$\mathrm{A}_{\mathrm{p}}$ : área do padrão interno;

$\mathrm{A}_{\mathrm{x}}$ : área do ácido graxo $\mathrm{x}$; 
$\mathrm{F}_{\text {cea }}$ : fator de conversão de metil éster para ácido graxo;

$\mathrm{F}_{\mathrm{ct}}$ : fator de correção (do detector de ionização chama) teórico do ácido graxo.

Os resultados obtidos através da equação 1 foram recalculados de $\mathrm{mg} / \mathrm{g}$ de lipídios totais para $\mathrm{mg} / 100 \mathrm{~g}$ de amostra desidratada.

Atividade antioxidante:

O extrato metanólico foi obtido por meio da percolação do solvente metanol através das amostras trituradas. $\mathrm{O}$ solvente foi removido em rotaevaporador rotatório sob pressão reduzida a $40^{\circ} \mathrm{C}$.

Realizou-se a investigação da atividade antioxidante das amostras através de reação com o radical DPPH (2,2difenil-1-picrilidrazila), Sigma, de acordo com El-Massary et al. (2002). O extrato obtido foi fracionado com solventes de diferentes polaridades, sendo eles, hexano, acetato de etila, 1-butanol e água. As frações foram coletadas separadamente e evaporadas sob pressão reduzida.

De cada fração, $20,0 \mathrm{mg}$ foram pesados e dissolvidos em 10,0mL de metanol, perfazendo uma concentração de 2 $\mathrm{mg} / \mathrm{mL}$. Volumes iniciais de 25, 50, 75 e $100 \mathrm{~mL}$ foram pipetados em cubetas. Ao abrigo da luz, adicionaram-se $2 \mathrm{~mL}$ da solução metanólica do radical DPPH 4,7\% (m/v), aguardando-se 30 min para a leitura da absorbância em $1=517 \mathrm{~nm}$. Para algumas amostras, os volumes iniciais foram alterados, para que o volume necessário para inibir $50 \%$ da absorção do radical DPPH (IC 50\%) fosse encontrado. As análises foram realizadas em triplicata, e os valores de absorvância determinados em espectrofotômetro (Cary 50-VARIAN), convertidos posteriormente em porcentagem de inibição do radical DPPH (\% AA) através da equação 2. Os valores de IC 50\% foram encontrados através de regressão linear.

$$
\% A A=100-\frac{\left(A b s_{\text {radical }}-A b s_{\text {amostra }}\right)}{A b s_{\text {radical }}}
$$

Onde:

$\mathrm{Abs}_{\text {radical }}=$ absorvância do radical DPPH

$\mathrm{Abs}_{\text {amostra }}=$ absorvância da amostra Análise Estatística:
Os dados foram comparados através da análise de variância (ANOVA) com nível de 5\% de significância, utilizando o programa STATISTICA (1995), versão 5.0. Os valores médios foram comparados pelo teste de Tukey.

\section{RESULTADOS E DISCUSSÃO}

Na composição centesimal das folhas (Tabela 1), não houve diferença significativa $(p>0,05)$ para os teores de umidade entre os três tempos analisados. O teor de proteína foi maior nos tempos de 40 dias $(30,53 \%)$ e 80 dias $(30,64 \%)$, sem diferença significativa entre eles. O maior valor de lipídios totais foi observado aos 40 dias (10,29\%). Os caules apresentaram menores teores de proteína, próximos a 7,22\%. Não houve diferença significativa para as porcentagens de cinzas e proteína.

Na Tabela 2 estão apresentados os principais ácidos graxos encontrados nas amostras analisadas. Quanto à composição dos ácidos graxos das folhas e caules, o majoritário foi o alfa-linolênico [LNA, 18:3 (n-3)], que apresentou maior porcentagem relativa entre todos os ácidos graxos identificados. Foram observadas as menores porcentagens relativas para os ácidos esteárico (18:0) e oléico (18:1n-9). Tal comportamento foi verificado para todos os tempos de cultivo avaliados (40, 80 e 120 dias).

Os caules e as folhas de linho (Tabela 2) apresentaram razões de ácidos graxos poli-insaturados/ ácidos graxos saturados (AGPI/AGS) superiores a 1,15, consideradas adequadas para alimentos, uma vez que o Departamento de Saúde e Seguridade Social da Inglaterra (HMSO, 1994) estabelece que valores menores a 0,45 são pouco aconselháveis para a saúde em relação às doenças cardíacas. As razões dos ácidos da série n-6 e n-3 (n-6/n-3), obtidas para caules e folhas ficaram entre 0,42 e 0,98, valores esses em conformidade com Enser et al. (1998), que estabelecem que essa razão seja no máximo 4,0. Portanto, as razões encontradas estão dentro dos níveis recomendados para o restabelecimento do equilíbrio na ingestão desses ácidos, na alimentação.

Tabela 1 - Composição centesimal das folhas e caules de linho, em diferentes estágios de desenvolvimento.

\begin{tabular}{ccccccc}
\hline \multirow{2}{*}{ Tempo } & \multicolumn{3}{c}{ Folhas } & \multicolumn{3}{c}{ Caules } \\
\cline { 2 - 7 } & 40 dias & 80 dias & 120 dias & 40 dias & 80 dias & 120 dias \\
\hline Umidade & $7,48^{\mathrm{a}} \pm 0,05$ & $7,52^{\mathrm{a}} \pm 0,03$ & $7,57^{\mathrm{a}} \pm 0,25$ & $9,79^{\mathrm{a}} \pm 0,27$ & $9,89^{\mathrm{a}} \pm 0,26$ & $5,23^{\mathrm{b}} \pm 0,06$ \\
Cinzas & $11,79^{\mathrm{a}} \pm 1,00$ & $11,98^{\mathrm{a}} \pm 0,09$ & $11,23^{\mathrm{b}} \pm 0,14$ & $6,89^{\mathrm{a}} \pm 0,34$ & $6,90^{\mathrm{a}} \pm 0,34$ & $5,85^{\mathrm{a}} \pm 0,04$ \\
Proteína & $30,53^{\mathrm{a}} \pm 0,45$ & $30,64^{\mathrm{a}} \pm 0,45$ & $26,13^{\mathrm{b}} \pm 0,31$ & $7,18^{\mathrm{a}} \pm 0,46$ & $7,24^{\mathrm{a}} \pm 0,47$ & $7,25^{\mathrm{a}} \pm 0,16$ \\
Lipídios totais & $10,29^{\mathrm{a}} \pm 0,57$ & $8,57^{\mathrm{ab}} \pm 0,16$ & $7,95^{\mathrm{b}} \pm 0,33$ & $4,91^{\mathrm{a}} \pm 0,22$ & $2,02^{\mathrm{b}} \pm 0,21$ & $2,42^{\mathrm{b}} \pm 0,10$ \\
\hline
\end{tabular}

Os resultados são médias \pm desvio padrão de análises em triplicatas. Letras diferentes na mesma linha (folhas e caules separadamente)
indicam diferenças significativas $(\mathrm{p}<0,05)$, pelo teste de Tukey. 


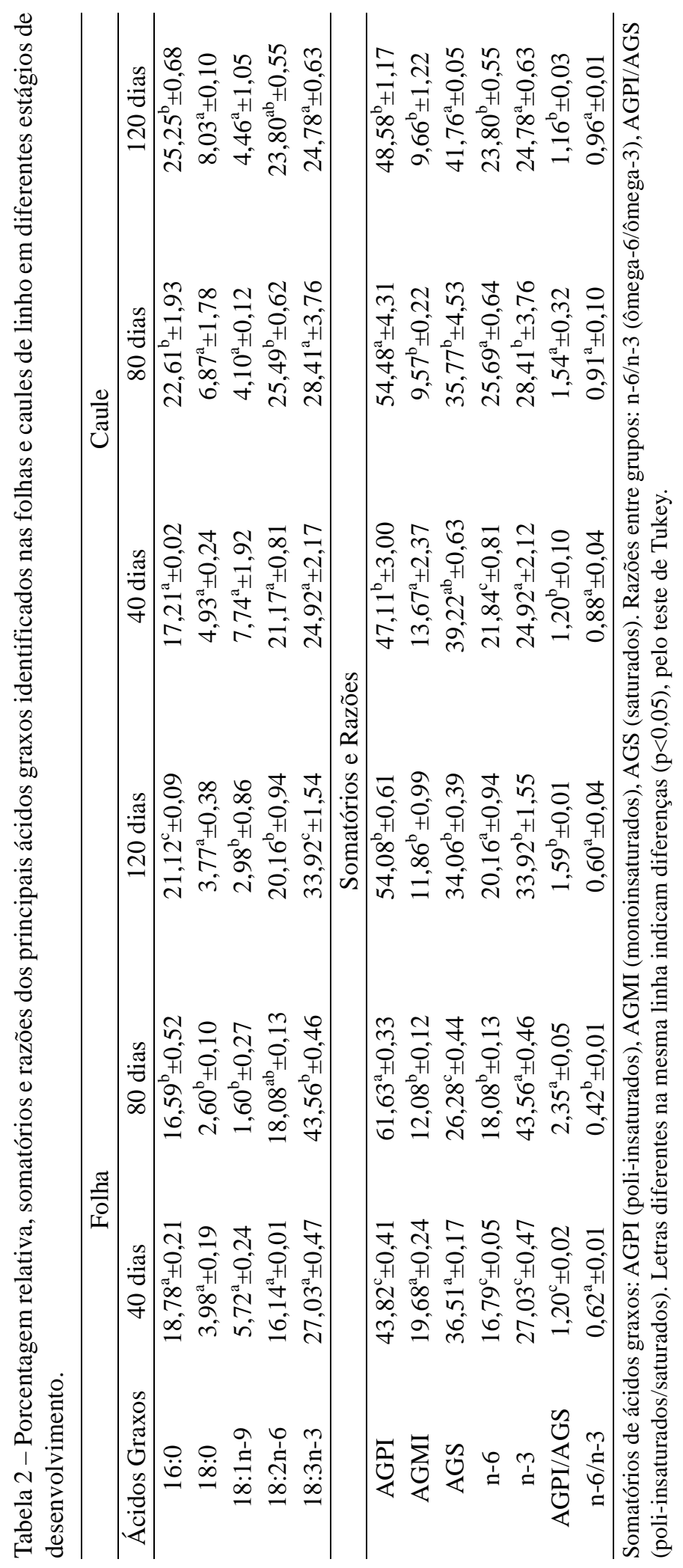

Ciênc. agrotec., Lavras, v. 34, n. 6, p. 1500-1506, nov./dez., 2010 
Como foi observada a predominância do LNA nas folhas e caules do linho, procedeu-se com a quantificação em $\mathrm{mg} / 100 \mathrm{~g}$ de amostra desidratada (Tabela 3), uma vez que esse ácido graxo é um constituinte nobre e está envolvido em processos metabólicos essenciais dos animais.

A Tabela 3 revela o comportamento da concentração de LNA ao longo do cultivo, que apresentou um aumento significativo aos 80 dias, tempo no qual foi atingida a máxima concentração de LNA nas folhas e caules, sendo $1.262,35 \mathrm{mg} / 100 \mathrm{~g}$ e $55,71 \mathrm{mg} / 100 \mathrm{~g}$ de amostra desidratada respectivamente, seguida de uma queda na concentração desse ácido graxo, no tempo de 120 dias.

Tabela 3 - Quantificação do LNA (mg/100g de amostra desidratada) nas folhas e caule de linho, colhidos em diferentes estágios de desenvolvimento.

\begin{tabular}{cccc}
\hline Tempo & 40 dias & 80 dias & 120 dias \\
\hline Folha & $398,69^{\mathrm{a}} \pm 2,11$ & $1.262,36^{\mathrm{b}} \pm 64,38$ & $663,91^{\mathrm{c}} \pm 12,37$ \\
Caule & $41,77^{\mathrm{a}} \pm 1,54$ & $55,71^{\mathrm{b}} \pm 1,11$ & $51,36^{\mathrm{b}} \pm 3,52$
\end{tabular}

Os resultados são médias \pm desvio padrão de análises em duplicatas. Letras diferentes na mesma linha indicam diferenças $(\mathrm{p}<0,05)$, pelo teste de Tukey.

Estudos de quantificação de LNA em alguns alimentos permitem compreender o alto teor encontrado nas folhas de linho colhidas aos 80 dias. Estudos realizados por Almeida et al. (2006), encontraram teores de 238,46 mg/100g de matéria seca para a Branquiária e 230,34 mg/100g de matéria seca para o Capim-estrela, ambas plantas forrageiras, valores inferiores aos encontrados para as folhas de linho nos diferentes tempos de colheita.

Durante o cultivo da planta, verificou-se um decréscimo no teor de LNA, após os 80 dias de cultivo, tempo no qual foi observada a formação das sementes. Isso pode ter ocorrido devido à transferência dos ácidos graxos LNA presentes nas folhas para as sementes, visto que, no tempo de 120 dias (completada a maturação das sementes) houve uma diminuição significativa de LNA nas folhas.

A atividade antioxidante das folhas do linho colhidas aos 80 dias apresentou valores satisfatórios, em especial para as frações butanólica e acetato, as quais inibiram $50 \%$ da absorbância do radical DPPH $\left(\mathrm{IC}_{50}\right)$ após a adição de aproximadamente $42 \mathrm{~mL}$ de seus extratos (Tabela 4). Para a fração aquosa, esse valor foi superior a $250 \mathrm{~mL}$, indicando portanto, atividade antioxidante inferior às frações acima citadas. Isso permite inferir que os compostos que atuam como antioxidantes nas folhas do linho têm polaridade intermediária, tal como o acetato de etila e álcool butanol-1.

Tabela 4 - Volume ( $\mu \mathrm{L}$ ) dos extratos obtidos das folhas de linho colhidas aos 80 dias, necessário para inibir 50\% do radical DPPH.

\begin{tabular}{cc}
\hline Fração & Volume $(\mu \mathrm{L})$ \\
\hline Hexanólica & $>500$ \\
Acetato & $41,99 \pm 1,027$ \\
Butanólica & $44,20 \pm 2,294$ \\
Metanólica & $117,18 \pm 0,790$ \\
Aquosa & $212,83 \pm 9,662$ \\
\hline
\end{tabular}

Os resultados são médias \pm desvio padrão de análises em triplicata.

A alta atividade antioxidante das folhas de 80 dias é condizente com os dados da literatura. LorencKukula et al. (2007) investigaram o conteúdo total de compostos fenólicos nas folhas e sementes da linhaça, encontrando valores superiores a $20 \mathrm{mg} / 100 \mathrm{~g}$ de extrato, para as folhas e $2,0 \mathrm{mg} / 100 \mathrm{~g}$ de extrato, para as sementes. Os compostos responsáveis pela atividade antioxidantes nesses alimentos são geralmente os flavonóides (Lorenc-Kukula et al., 2007), cuja estrutura contempla compostos fenólicos, que através da estabilização dos radicais livres, inibe a peroxidação lipídica enzimática e não enzimática nos alimentos (Boroski et al., 2008). Tem crescido o número de trabalhos que investigam a ação de compostos que evitam a oxidação, tais como as lignanas, abundantemente encontradas na linhaça (Gurib-Fakim, 2006), assim como métodos analíticos para obtê-los das mais diferentes matrizes alimentares (Buranov \& Mazza, 2008).

\section{CONCLUSÕES}

As folhas de linho se apresentaram como uma valiosa fonte de proteínas, além de terem fornecido elevadas concentrações de LNA em sua fração lipídica, em 80 dias de cultivo após a germinação.

A composição centesimal e teor de LNA nos caules mantiveram-se constantes. As frações butanólica e acetato das folhas colhidas no tempo de 80 dias apresentaram alta atividade antioxidante, evidenciando o potencial nutritivo e ação antioxidante das folhas de linho para alimentação animal e humana. 


\section{REFERÊNCIAS BIBLIOGRÁFICAS}

ALMEIDA, V.V.; BANI, F.A.; STEVANATO, F.B.; PRADO, I.N.; MATSUSHITA, M.; VISENTAINER, J.V. A influência da composição lipídica de capins sobre a formação de CLA em ruminantes (conjugated linoleic acid): aspectos gerais. Revista Nacional da Carne, São Paulo, n.355, p.111-112, set. 2006.

BLIGH, E.G.; DYER, W.J. A rapid method of total lipid extraction and purification. Canadian Journal Biochemistry Physiology, Ontario, v.37, p.911-917, ago. 1959.

BOROSKI, M.; PERINI, J.A.L.; ROTTA, E.M.; MATSUSHITA, M.; SOUZA, N.E.; VISENTAINER, J.V. Efeito de condimentos e composição em ácidos graxos em carne de frango temperada. Revista Nacional da Carne, São Paulo, n.382, p.72-77, dez. 2008.

BURANOV A.U.; MAZZA, G. Lignin in straw of herbaceous crops. Industrial Crops And Products, London, v.28, n.3, p.237-259, nov. 2008.

CARTER, J.F. Potential of flaxseed and flaxseed oil in baked goods and other products in human nutrition. Cereal Foods World, Minneapolis, v.38, n.10, p.753-59, 1993.

CHU, Y.H.; CHANG, C.L.; HSU, H.F. Flavonoid content of several vegetables and their antioxidant activity. Journal of the Science of Food and Agriculture, London, v.80, n.5, p.561-566, Apr. 2000.

EL-MASSARY, K.F.E.; EL-GHORAB,A.H.; FAROUK, A. Antioxidant activity and volatile components pf Egyptian Artemisia judaica L. Food Chemistry, Barking, v.79, n.3, p.331-336, Mar. 2002.

ENSER, M.; HALLETT, K.G.; FURSEY, A.J.; WOOD, J.D.; HARRINGTON, G. Fatty acid content and composition of UK beef and lamb muscle in relation to production system and implications for human nutrition. Meat Science, New York, v.49, p.329-341, July 1998.

GURIB-FAKIM, A.A. Medicinal plants: traditions of yesterday and drugs of tomorrow. Molecular Aspects of Medicine, New York, v.27, n.1, p.1-9, Feb. 2006.

HARTMAN, L.; LAGO, R.C.A. Rapid preparation of fatty acid methyl esters from lipids. Laboratory Practice, London, v.22, n.8, p.475-476, 1973.
HMSO. Nutritional aspects of cardiovascular disease: department of health. Report in health and Social Subjects, London, n.46, p.178, 1994.

JOSEPH, J.D.; ACKMAN, R.G. Capillary column gas chromatography method for analysis of encapsulated fish oil and fish oil ethyl esters: collaborative study. Journal Association of Official Analytical Chemists, Gaithersburg, v.75, n.3, p.488-506, 1992.

KISS, J.; SOUZA, E. Linho: flores da tradição. Revista Globo Rural, São Paulo, v.15, n.171, p.36-41, jan. 2000

LIYANA-PATHIRANA, C.M.; SHAHIDI, F. Antioxidant properties of comercial soft and hard Winter wheats (Triticum aestivum $L$.) and their milling fractions. Journal of the Science of Food and Agriculture, London, v.86, n.3, p.477-485, Feb. 2006.

LORENC-KUKULA, K.; WROBEL-KWIATKOWSKAA, M.; STARZYCKIC, M.; SZOPAA, J. Engineering flax with increased flavonoid content and thus Fusarium resistance. Physiological and Molecular Plant Pathology, London, v.70, n.1/3, p.38-48, Jan./Mar. 2007.

MARTIN, C.A.; ALMEIDA, V.V.; RUIZ, M R.; VISENTAINER, J.E.L.; MATSUSHITA, M.; SOUZA, N.E.; VISENTAINER, J.V. Acidos graxos poliinsaturados ômega-3 e ômega-6: importância e ocorrência em alimentos. Revista de Nutrição, Campinas, v.19, n.6, p.761-770, nov./dez. 2006.

MORRIS, D.H. Flax: a health and nutrition primer. 4.ed. Ottawa, 2007. Disponível em: <hitp://Www.flaxcouncil.ca ienglish/index.jsp? p=nutrition2 2 mp=nutrition em: 22 dez. 2008

MOURE, A.; CRUZ, J.M.; FRANCO, D.; DOMINGUEZ, J.M.; SINEIRO, J.; DOMINGUEZ, H.; NIÑEZ, M.J.; PARAJÓ, J.C. Natural antioxidants from residual sources. Food Chemistry, Barking, v.72, n.2, p.145-171, Feb. 2001

PRADO, I.N.; ROTTA, P.P.; VISENTAINER, J.V.; MOLETTA, J.L.; PEROTTO, D. Carcass characteristics and chemical composition of the Longissimus muscle of crossbred bulls (Bostaurus indicus vs Bos taurus taurus) finished in feedlot. Journal of Animal and Feed Sciences, Jablonna, v.17, p.295-306, 2008. 
SILVA, D.C.; SANTOS, G.T.; PINTRO, P.T.M.; BOATTO, D.A.; VISENTAINER, J.V.; PETIT, H.V. Butter quality of Holstein cows fed whole or ground flaxseed with or without monensin. In: International Workshop on the biology of lactation in farm animals, 8., 2006,

Pirassununga, SP. Revista Ciência Veterinária, Pirassununga, p.30-31, 2006.

SIMOPOULOS, A.P. Omega 3 fatty acids in health and disease and is growth and development. American Journal of Clinical Nutrition, Washington, v.54, n.3, p.438-63, Sept. 1991.

SOUZA, N.E.; STEVANATO, F.B.; GARCIA, E.E.; VISENTAINER, J.E.L.; ZARA, R.F.; VISENTAINER, J.V. Supplemental dietary flaxseed oil affects both neural and phospholipid fatty acids in culture tilapia. European Journal of Lipid Science and Technology, Weinheim, v.110, n.8, p.671-786, Aug. 2008.
STATISTICA. Statistica 5.1 softwer. Tucks, 1995.

VISENTAINER, J.V.; CARVALHO, P.O.; IKEGAKI, M.; PARK, Y.K. Determinação de ácido eicosapentaenóico (EPA) e ácido docosaexaenóico (DHA) em peixes marinhos da costa brasileira. Ciência e Tecnologia de Alimentos, Campinas, v.20, n.1, p.90-93, maio/ago. 2000.

VISENTAINER, J.V.; FRANCO, M.R.B. Ácidos graxos em óleos e gorduras: identificação e quantificação. São Paulo: Varela, 2006. 120p.

VISENTAINER, J.V.; SOUZA, N.E.; MATSUSHITA, M.; HAYASHI, C.; FRANCO, M.R.B. Influence of diets enriched with flaxseed oil on the alfa-linolenic, eicosapentaenoic and docosahexaenoic fatty acid in Nile tilapia (Oreochromis niloticus). Food Chemistry, Barking, v.9, n 4, p.557-560, May 2005. 